\title{
Gas Concentration Prediction Based on the Measured Data of a Coal Mine Rescue Robot
}

\author{
Xiliang Ma and Hua Zhu \\ School of Mechanical and Electrical Engineering, China University of Mining and Technology, Xuzhou, Jiangsu 221116, China \\ Correspondence should be addressed to Xiliang Ma; xlma818@sina.com
}

Received 26 November 2015; Revised 24 February 2016; Accepted 14 March 2016

Academic Editor: Giovanni Muscato

Copyright (C) 2016 X. Ma and H. Zhu. This is an open access article distributed under the Creative Commons Attribution License, which permits unrestricted use, distribution, and reproduction in any medium, provided the original work is properly cited.

The coal mine environment is complex and dangerous after gas accident; then a timely and effective rescue and relief work is necessary. Hence prediction of gas concentration in front of coal mine rescue robot is an important significance to ensure that the coal mine rescue robot carries out the exploration and search and rescue mission. In this paper, a gray neural network is proposed to predict the gas concentration 10 meters in front of the coal mine rescue robot based on the gas concentration, temperature, and wind speed of the current position and 1 meter in front. Subsequently the quantum genetic algorithm optimization gray neural network parameters of the gas concentration prediction method are proposed to get more accurate prediction of the gas concentration in the roadway. Experimental results show that a gray neural network optimized by the quantum genetic algorithm is more accurate for predicting the gas concentration. The overall prediction error is $9.12 \%$, and the largest forecasting error is $11.36 \%$; compared with gray neural network, the gas concentration prediction error increases by $55.23 \%$. This means that the proposed method can better allow the coal mine rescue robot to accurately predict the gas concentration in the coal mine roadway.

\section{Introduction}

Coal mine accidents have occurred frequently in our country. Coal mine accidents caused by a gas outburst, gas explosion, and gas suffocation accounted for $70 \% \sim 80 \%$ of all coal mine accidents [1], which not only cause great losses to the coal mining enterprises but also bring huge economic losses and psychological harm to the enterprise employees, family members, and the country. After a coal mine accident, the timeliness of an emergency rescue is very important. Rescue and relief workers have a small window of opportunity to enter the underground scene of the accident for rescue purposes. However, a complex and dangerous coal mine environment is a great security threat for rescue workers. With the development of science and technology, the mine rescue robot can be used for coal mine disaster relief to detect the situation within the disaster areas, to find personnel in distress, and to provide decision-making support for the rescue.
Gas disaster accidents often destroy roadway ventilation facilities, damage ventilation systems, and cause methane to flow from the coal wall and the goaf, most likely forming gas accumulation in the disaster areas. Furthermore, there is the risk of elevated temperature, coal dust, and other flammable and explosive gases produced from coal dry distillation, further increasing the risk of gas explosion. Not extinguishing the fire or new flames produced by secondary factors may cause a secondary gas explosion at any time. To ensure that the coal mine rescue robot can perform the detection and search and rescue mission, measures must be taken to avoid secondary disasters such as gas explosion caused by concentration. Therefore, predicting gas concentrations early has very important significance.

A comprehensive analysis of domestic and foreign research on gas concentration forecast shows that, at present, the research mainly focuses on the prediction of gas emissions, gas concentration prediction algorithms or methods, and so on. Özgen Karacan [2] proposes a principle component analysis and artificial neural network-based approach 
to predict the ventilation methane emission rates of US longwall mines. Runqiua et al. [3] put forward a prediction gas emission method based on self-organizing data mining, and $\mathrm{Hu}$ et al. [4] established prediction models of the coalbed methane content using quantification theory based on the limited data obtained from the exploratory bores. The prediction method and algorithm of the gas concentration are compared with the prediction model [5] or an improved prediction model [6] to improve the accuracy of prediction. By using an effective prediction method or algorithm [7-9] to validate the practicality of the prediction [10] and using the combination forecasting method or algorithm [11-14] to accurately predict the concentration range, the trend of the gas concentration can be tracked effectively. However, there are some problems in these studies. Predicting the emission quantity is mostly based on data statistics or data mining. Searching for a gas change law and predicting the amount of gas emission in a mine or a range often require a large amount of data. Sometimes, data from the past few decades are needed, resulting in a large amount of forecast work. Furthermore, among the research in the gas concentration prediction method or algorithm, the accuracy and effectiveness of the method or algorithm are of greater concern, and the practicability of the algorithm is seldom considered. If the gray system and neural network are combined to form the gray neural network, it can take full advantage of the gray system's data modeling and the neural network's nonlinear mapping features. With the help of the gray mode, the gray neural network uses new data after the accumulation of new data to model. To a certain extent, this process weakens the randomness of the original data, and it is easy to find the transformation of the data. This approach is suitable when partial information is known, some information is unknown, there is a small sample, there is poor information, or there is uncertainty in the system. The establishment of a gray neural network can solve the problem of insufficient data quantity for gas concentration, but it also has its own inherent defects, such as randomness in the weight and threshold in the initialization process, falling into the local optimum during network evolution, and the inability to further modify the relevant parameters. Therefore, to improve the accuracy and applicability of gray neural network forecasts, the gray neural network is combined with the optimization algorithm. Currently, more common optimization algorithms include the particle swarm algorithm, ant colony algorithm, and genetic algorithm. The particle swarm optimization algorithm's main problem is that it is easy to produce premature convergence (especially when dealing with complex multimodal search problems) and poor local search optimization capabilities. The PSO algorithm is at the local minimum, which is mainly due to the loss of diversity in the search space. The ant colony algorithm is a typical probabilistic algorithm. The parameter settings in the ant colony algorithm are usually determined by experiments, which are closely related to human experience, and it is difficult to optimize the performance of the algorithm. The genetic algorithm can find the optimal solution of the problem using probability, but it does not take advantage of the excellent evolution immature subset of the information provided in the optimization process. In addition, there are slow convergence and premature convergence defects. The quantum genetic algorithm is introduced into the genetic optimization algorithm, which makes the genetic operation more effective. A quantum bit was introduced into the chromosome coding in the quantum state vector based on the narration. Thus, a chromosome can express the superposition of multiple states, reduce the size of the population, and increase the diversity of the population. At the same time, the application of the concept of a quantum gate can promote the convergence of the algorithm, and the quantum gate mutation operation can be used.

The phase characteristics of quantum entanglement can break the traditional genetic algorithm to produce several individual concept hybrid offspring. Using the quantum entanglement of quantum hybrid design can exchange information in the entire population, making it easy to find great individual populations. Hybrid quantum entanglement with quantum information can be communicated throughout the population, making it easy to find great individual populations.

This paper combines the actual work requirements of the coal mine rescue robots with a small amount of measurement data of the robot, and the factors affecting the gas concentration (such as temperature, wind speed, and distance) are considered. The gas concentration 10 meters in front of the coal mine rescue robot is predicted using the gray neural network variation for eigenvalues in uncertain systems. The prediction accuracy of the gray neural network is improved by optimizing the quantum genetic algorithm parameters of the gray neural network. The safety of the coal mine rescue robot's task execution is ensured by avoiding gas explosion accidents because of either the coal mine rescue robot itself or the ground friction sparks. This provides an effective basis for the rescue decision.

\section{Model Establishment}

2.1. Gray Neural Network. Grey system theory is founded by Professor Deng Julong in 1982, an expansion theory from the method of white-black-gray box, which is a new research method for the issue with a few new original data and uncertain features. A gray neural network is the combination of gray theory and a neural network, which can predict the development and change of the characteristic value of the uncertain system. The original sequence of the system $x_{t}^{(0)}$ $(t=1,2, \ldots, N-1)$, after a cumulative sequence gives $x_{t}^{(1)}$, and its exponential growth can be used as a function or differential equation for data fitting and prediction. To facilitate representation, redefine the original sequence $x(t)$ after sequence $x_{t}^{1}$ is represented as a cumulative sequence, and the result is expressed as a forecast.

The differential predictive model of the parameters in a gray neural network is

$$
\frac{d y}{d t}+a y_{1}=b_{1} y_{2}+b_{2} y_{3}+\cdots+b_{n-1} y_{n}
$$

where $y_{1}, y_{2}, \ldots, y_{n}$ are the input parameters for the system; $y_{1}$ is the system output parameter; and $a, b_{1}, b_{2}, \ldots, b_{n}$ are the 


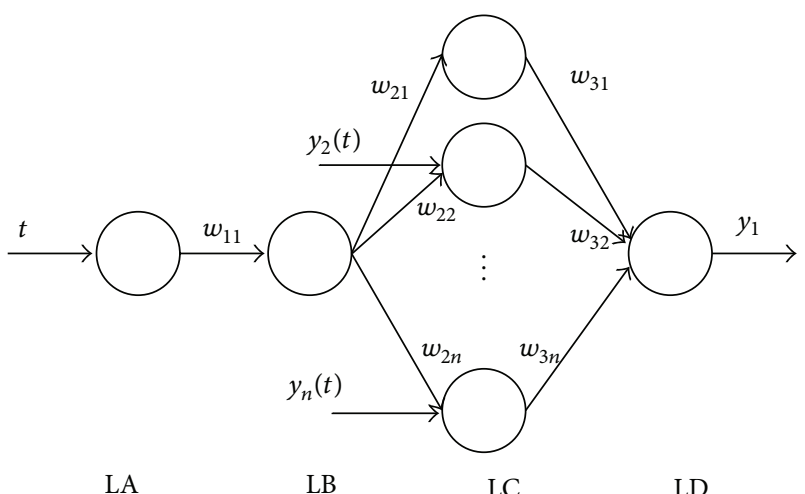

FIGURE 1: Gray neural network structure topology.

coefficient differential equations. The time response formula of (1) is

$z(t)$

$$
\begin{aligned}
& =\left(y_{1}(0)-\frac{b_{1}}{a} y_{2}(t)-\frac{b_{2}}{a} y_{3}(t)-\cdots-\frac{b_{n-1}}{a} y_{n}(t)\right) \\
& \cdot e^{-a t}+\frac{b_{1}}{a} y_{2}(t)+\frac{b_{2}}{a} y_{3}(t)+\cdots+\frac{b_{n-1}}{a} y_{n}(t) .
\end{aligned}
$$

Let $d=\left(b_{1} / a\right) y_{2}(t)-\left(b_{2} / a\right) y_{3}(t)-\cdots-\left(b_{n-1} / a\right) y_{n}(t)$. Equation (2) can be simplified to

$z(t)$

$$
\begin{aligned}
= & \left(\left(y_{1}(0)-d\right) \cdot \frac{e^{-a t}}{e^{-a t}+1}+d \cdot \frac{1}{e^{-a t}+1}\right) \\
& \cdot\left(e^{-a t}+1\right) \\
= & \left(\left(y_{1}(0)-d\right) \cdot\left(1-\frac{e^{-a t}}{e^{-a t}+1}\right)+d \cdot \frac{1}{e^{-a t}+1}\right) \\
& \cdot\left(e^{-a t}+1\right) \\
= & \left(\left(y_{1}(0)-d\right)-y_{1}(0) \cdot \frac{e^{-a t}}{e^{-a t}+1}+2 d \cdot \frac{1}{e^{-a t}+1}\right) \\
& \cdot\left(e^{-a t}+1\right) .
\end{aligned}
$$

Mapping (3) to the BP neural network gives the gray neural network with $N$ input parameters and 1 output parameter, and the network topology is shown in Figure 1.

In the graph, $t$ is the sequence number, and $y_{2}(t), y_{3}(t)$, $\ldots, y_{n}(t)$ are the network input parameters, $w_{21}, w_{22}, \ldots, w_{2 n}$ and $w_{31}, w_{32}, \ldots, w_{3 n}$ are the network weight values, and $y_{1}$ is the network forecast value. LA, LB, LC, and LD represent the four layers in the gray neural network.
Let $2 b_{1} / a=u_{1}, 2 b_{2} / a=u_{2}, \ldots, 2 b_{n-1} / a=u_{n-1}$, so the network weights can be expressed as follows:

$$
\begin{aligned}
& w_{11}=a ; \\
& w_{21}=-y_{1}(0) ; \\
& w_{22}=u_{1}, \ldots, w_{2 n}=u_{n-1} ; \\
& w_{31}=w_{32}=\cdots=w_{3 n}=1+e^{-a t} .
\end{aligned}
$$

The LD layer output node threshold is

$$
\theta=\left(1+e^{-a t}\right)\left(d-y_{1}(0)\right)
$$

The gray neural network training process is as follows:

(1) Initialize the gray neural network and the parameters.

(2) Each output is calculated for each input sample:

LA layer: $a=w_{11} t$.

LB layer: $b=f\left(w_{11} t\right)=1 /\left(1+e^{-w_{11} t}\right)$.

LC layer: $c_{1}=b w_{21}, c_{2}=y_{2}(t) b w_{22}, \ldots, c_{n}=$ $y_{n}(t) b w_{2 n}$.

LD layer: $d=w_{31} c_{1}+w_{32} c_{2}+\cdots+w_{3 n} c_{n}-\theta_{y 1}$.

(3) Calculate the error of the predicted output and the actual value, and the error formula of each layer is as follows:

LD layer: $\delta=d-y_{1}(t)$.

LC layer: $\delta_{1}=\delta\left(1+e^{-w_{11} t}\right), \delta_{2}=\delta\left(1+e^{-w_{11} t}\right), \ldots, \delta_{n}=$ $\delta\left(1+e^{-w_{11} t}\right)$.

LB layer: $\delta_{n+1}=\left(1 /\left(1+e^{-w_{11} t}\right)\right)(1-1 /(1+$ $\left.\left.e^{-w_{11} t}\right)\right)\left(w_{21} \delta_{1}+w_{22} \delta_{2}+\cdots+w_{2 n} \delta_{n}\right)$.

(4) Adjust the threshold formula in accordance with the following error:

LB to LC layer: $w_{21}=-y_{1}(0), w_{22}=w_{22}-u_{1} \delta_{2} b$, $\ldots, w_{2 n}=w_{2 n} u_{n-1} \delta_{n}$.

LA to LB layer: $w_{11}=w_{11}+a t \delta_{n+1}$;

Adjust the threshold: $\theta=\left(1+e^{-w_{11} t}\right)\left(\left(w_{22} / 2\right) y_{2}(t)+\right.$ $\left.\left(w_{23} / 2\right) y_{3}(t)+\cdots+\left(w_{2 n} / 2\right) y_{n}(t)-y_{1}(0)\right)$.

(5) Determine whether the set error is reached. If it is reached, the training is over, and if not, return to step (2)

\subsection{Quantum Genetic Algorithm}

2.2.1. Quantum Genetic Algorithm Coding. The common coding methods of the quantum genetic evolutionary algorithm are binary coding, decimal coding, and symbol coding. A novel coding scheme based on quantum bit is used in the quantum evolutionary strategy. Its most essential feature is the full use of superposition and the coherence of quantum states, as well as the entangled states of the quantum bits. The smallest unit of information is a quantum bit, or a qubit. In the quantum genetic algorithm, a qubit may be in $|1\rangle$ or $|0\rangle$, in an intermediate state of $|1\rangle$ and $|0\rangle$, and in different 
superpositions of $|1\rangle$ and $|0\rangle$. Therefore, a qubit state is expressed as follows:

$$
|\psi\rangle=\alpha|0\rangle+\beta|1\rangle
$$

In the formula, $\alpha$ and $\beta$ can be complex, and they represent the probability amplitude of the corresponding state and satisfy the condition $\alpha^{2}+\beta^{2}=1$.

A system with $\mathrm{m}$ quantum bits can be expressed as $2^{m}$ states, namely,

$$
\left[\begin{array}{l}
\alpha_{1}, \alpha_{2}, \ldots, \alpha_{m} \\
\beta_{1}, \beta_{2}, \ldots, \beta_{m}
\end{array}\right] .
$$

In the formula, $\alpha_{i}^{2}+\beta_{i}^{2}=1, i=1,2, \ldots, m$.

2.2.2. Quantum Variation. By the basic assumptions of quantum mechanics, $n$-bit quantum registers can be in $2^{n}$ ground state of coherent superposition state $|\psi\rangle$; that is to say, it can simultaneously represent the $2^{n}$ number. The relationship between superposition state and ground state can be expressed as $|\psi\rangle=\sum_{i} C_{i}\left|\psi_{i}\right\rangle$, where $C_{i}$ is the probability amplitude of the state $\psi_{i}$, and $\left|C_{i}\right|^{2}$ express the probability of $\psi$ collapse to the ground state $\psi_{i}$, when subjected to quantum computing systems and measuring instruments observation entangled.

Quantum coherence [15] is an important mechanism for quantum computing, using a special link between the quantum states; other quantum states can be launched from one or more quantum states. One of the main principles of quantum computing is to make each ground state of superposition state change the relative phase between them through quantum gate interference effect. For example, superposition state and quantum gate are given as follows:

$$
\begin{aligned}
\left|\psi^{\prime}\right\rangle & =\frac{2}{\sqrt{5}}|0\rangle+\frac{1}{\sqrt{5}}|1\rangle=\frac{1}{\sqrt{5}}\left(\begin{array}{l}
2 \\
1
\end{array}\right), \\
U & =\frac{1}{\sqrt{2}}\left(\begin{array}{cc}
1 & 1 \\
1 & -1
\end{array}\right) .
\end{aligned}
$$

Quantum gate action in superposition state as a result is as follows:

$$
\left|\psi^{\prime}\right\rangle=\frac{3}{\sqrt{10}}|0\rangle+\frac{1}{\sqrt{10}}|1\rangle \text {. }
$$

From (9) it can be seen that the probability amplitude of ground state $|1\rangle$ decreases, while the probability amplitude of $|0\rangle$ increases. The quantum system is coherent when its ground state is in linear superposition state. The linear superposition of coherent system will disappear when interacting with the surrounding environment, the process called decoherence.

Another important mechanism for quantum computing is quantum entanglement. The so-called entanglement refers to some states existing in two interaction subsystems; it cannot be represented as tensor product of two subsystems' state but rather as some entanglement form of the subsystem
TABLE 1: Adjustment strategy of the quantum rotation gate.

\begin{tabular}{cccccccc}
\hline$x_{i}$ & $b_{i}$ & $f(x)<f(b)$ & $\theta$ & \multicolumn{4}{c}{$S\left(\alpha_{i}, \beta_{i}\right)$} \\
\hline 0 & 0 & $\mathrm{~F}$ & 0 & 0 & 0 & 0 & 0 \\
0 & 0 & $\mathrm{~T}$ & 0 & 0 & 0 & 0 & 0 \\
0 & 1 & $\mathrm{~F}$ & 0 & 0 & 0 & 0 & 0 \\
0 & 1 & $\mathrm{~T}$ & $0.05 \pi$ & -1 & +1 & \pm 1 & 0 \\
1 & 0 & $\mathrm{~F}$ & $0.01 \pi$ & -1 & +1 & \pm 1 & 0 \\
1 & 0 & $\mathrm{~T}$ & $0.025 \pi$ & +1 & -1 & 0 & \pm 1 \\
1 & 1 & $\mathrm{~F}$ & $0.005 \pi$ & +1 & -1 & 0 & \pm 1 \\
1 & 1 & $\mathrm{~T}$ & $0.025 \pi$ & +1 & -1 & 0 & \pm 1 \\
\hline
\end{tabular}

state. Some certain state of an entangled quantum system does not correspond to the state of the subsystem; certain correlation exists between the various subsystems. For example, $|00\rangle+|11\rangle$ cannot be expressed as a tensor product of two subsystems' state. Otherwise, there must be four numbers $a_{1}$, $a_{2}, b_{1}$, and $b_{2}$, satisfying

$$
\left(a_{1}|0\rangle+b_{1}|1\rangle\right) \otimes\left(a_{2}|0\rangle+b_{2}|1\rangle\right)=|00\rangle+|11\rangle .
$$

Furthermore,

$$
\begin{aligned}
& \left(a_{1}|0\rangle+b_{1}|1\rangle\right) \otimes\left(a_{2}|0\rangle+b_{2}|1\rangle\right) \\
& \quad=a_{1} a_{2}|00\rangle+a_{1} b_{2}|01\rangle+a_{2} b_{1}|10\rangle+b_{1} b_{2}|11\rangle .
\end{aligned}
$$

Suppose that (11) holds; then get $a_{1} b_{2}=0$ and $a_{2} b_{1}=0$, which could indicate $a_{1} a_{2}=0$ or $b_{1} b_{2}=0$. Obviously, (10) and (11) should not be established at the same time because of its self-contradiction. Therefore, in the quantum system, the entire system cannot use quantum state of each qubit but is described by entangled quantum states. The operation of a few of entangled qubits will not only change the state of the qubits but also change the other qubits in which they are entangled.

The most essential feature of the quantum genetic algorithm is making full use of the superposition and coherence of quantum states, as well as the entangled states of the quantum bits. The quantum unitary transformation process applies the information processing process to the classical genetic algorithm. Using the quantum bit coding chromosome, the evolution of the population is realized through quantum mutation. A rotating quantum gate is a type of quantum gate that evolves by the rotation of the quantum gates. The information of the best individual can be added to the mutation to guide the evolution and speed up the convergence of the algorithm. The adjustment operation of the quantum rotation gate is shown in Table 1.

$x_{i}$ and $b_{i}$, respectively, refer to solution $x$ and binary bits corresponding to the $i$ th quantum bit of the current best individual $B . f(x)$ is the fitness function. The variation of the rotation angle controls the convergence speed of the algorithm for the direction of the rotation angle to ensure the algorithm's convergence.

The rotational quantum gate ensures that the algorithm quickly converges into a chromosome with higher fitness. When $x_{i}=0, b_{i}=0, f(x) \geq f(b)$, to make the 


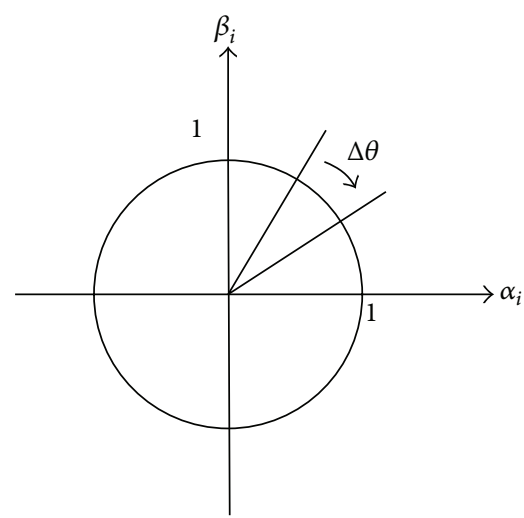

FIGURE 2: Quantum spin in the revolving door program.

TABLE 2: Chromosomes before cross-interference.

\begin{tabular}{llllll}
\hline Chromosome & \multicolumn{5}{c}{ Genetic factor } \\
\hline 1 & $\mathrm{~A}(1)$ & $\mathrm{A}(2)$ & $\mathrm{A}(3)$ & $\mathrm{A}(4)$ & $\mathrm{A}(5)$ \\
2 & $\mathrm{~B}(1)$ & $\mathrm{B}(2)$ & $\mathrm{B}(3)$ & $\mathrm{B}(4)$ & $\mathrm{B}(5)$ \\
3 & $\mathrm{C}(1)$ & $\mathrm{C}(2)$ & $\mathrm{C}(3)$ & $\mathrm{C}(4)$ & $\mathrm{C}(5)$ \\
4 & $\mathrm{D}(1)$ & $\mathrm{D}(2)$ & $\mathrm{D}(3)$ & $\mathrm{D}(4)$ & $\mathrm{D}(5)$ \\
5 & $\mathrm{E}(1)$ & $\mathrm{E}(2)$ & $\mathrm{E}(3)$ & $\mathrm{E}(4)$ & $\mathrm{E}(5)$ \\
\hline
\end{tabular}

current converge to a higher degree of adaptation of the chromosome, $\left|\alpha_{i}^{t}\right|^{2}$ should be larger. Therefore, if $\left(\alpha_{i}, \beta_{i}\right)$ are in the first and fourth quadrants, $\theta$ should rotate clockwise, and if $\left(\alpha_{i}, \beta_{i}\right)$ are in the second and third quadrants, $\theta$ should rotate counterclockwise, as shown in Figure 2.

2.2.3. Quantum Cross. The optimal value is often not precisely known in the actual data optimization process. The optimal value established in the optimization process often already counts the extreme value out, thus causing the system to fall into the local optimum. Quantum crossover is an effective method to prevent the system from the local optimal solution. The quantum crossover evolution strategy is an effective means to prevent the system from falling into local optima. In the genetic algorithm, the crossover is a means of searching the optimal solution, and its role is to achieve structural information exchange between individuals. According to the characteristics of the prediction of the gas concentration, the method of cross-interference is a full cross-interference in this paper [16], which uses quantum coherence properties and constructed a new crossover for ordinary chromosome. All the chromosomes in the population are involved in the crossover operation. Suppose that there are initially five chromosomes and that each chromosome has five genes, as shown in Table 2. Take chromosome 1 from the first genetic factor, chromosome 2 from the second genetic factor, chromosome 3 from the third genetic factor, chromosome 4 from the fourth genetic factor, and chromosome 5 from the fifth genetic factor. After the whole interference crossover, the first chromosome forms a new chromosome: $\mathrm{A}(1)-\mathrm{B}(2)-\mathrm{C}(3)-\mathrm{D}(4)-\mathrm{E}(5)$, as shown in Table 3.
TABLE 3: Chromosomes after cross-interference.

\begin{tabular}{llllll}
\hline Chromosome & \multicolumn{5}{c}{ Genetic factor } \\
\hline 1 & $\mathrm{~A}(1)$ & $\mathrm{B}(2)$ & $\mathrm{C}(3)$ & $\mathrm{D}(4)$ & $\mathrm{E}(5)$ \\
2 & $\mathrm{~B}(1)$ & $\mathrm{C}(2)$ & $\mathrm{D}(3)$ & $\mathrm{E}(4)$ & $\mathrm{A}(5)$ \\
3 & $\mathrm{C}(1)$ & $\mathrm{D}(2)$ & $\mathrm{A}(3)$ & $\mathrm{A}(4)$ & $\mathrm{B}(5)$ \\
4 & $\mathrm{D}(1)$ & $\mathrm{E}(2)$ & $\mathrm{B}(3)$ & $\mathrm{B}(4)$ & $\mathrm{C}(5)$ \\
5 & $\mathrm{E}(1)$ & $\mathrm{A}(2)$ & $\mathrm{C}(3)$ & $\mathrm{C}(4)$ & $\mathrm{D}(5)$ \\
\hline
\end{tabular}

The purpose of this cross-interference is to fully exploit the information of all chromosomes in the population to improve the locality and one-sidedness. This method can also be applied to the evolution of the common chromosome. When there is premature population evolution, it can process new impetus into evolutionary processes by producing a new individual.

2.2.4. Fitness Function. A fitness function is the evaluation criterion of the algorithm to the individual's "good and bad," and its value is positive.

This paper intends to use the quantum genetic algorithm optimization gray neural network parameters to predict the roadway gas concentration. Therefore, the error of the gray neural network's prediction value of the training data and its actual value are used as the fitness function as follows:

$$
Q=\operatorname{sum}\left(\left|O_{P i}-O_{R i}\right|\right) \text {. }
$$

$O_{P i}$ is used to predict the gas concentration, and $O_{R i}$ is used for the actual gas concentration value.

\section{Experimental Analysis}

3.1. Experimental Process. The coal mine rescue robot and the related sensor position are shown in Figure 3. The mine rescue robot is the dual arm robot. Position 1 is connected internally with the photoelectric encoder to detect the robot's walking distance and moving speed. Position 2 is the robot's power supply and drive unit. Position 3 is the control unit of the robot, where the instruction of the host computer is processed and the robot's state and the environmental information are collected and processed. Position 4 is a multiparameter detector, which can simultaneously detect methane, carbon dioxide, carbon monoxide, oxygen, temperature, humidity, and other parameters. Position 5 is the wind speed and direction sensors.

The gas concentration prediction experiments based on the coal mine rescue robot-measured data are carried out in the coal mine tunnel. The coal mine rescue robot's walking speed is $1 \mathrm{~m} / \mathrm{s}$, and after every 0.5 meters, all sensor data are uploaded (but also according to instructions to the upload data). The core of the robot control unit is the embedded industrial computer, which is powerful at processing data.

The main factors influencing the gas flow are air flow motion, diffusion motion caused by concentration gradient, and floating motion caused by density difference, and so on, while in the tunnel it is mainly affected by the air flow motion and diffusion motion. Gas diffusion is the migration form 


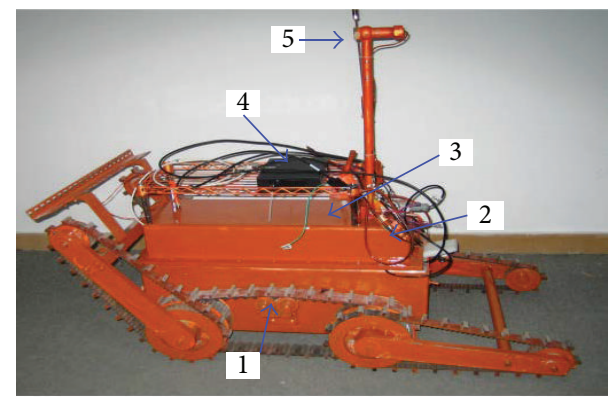

FIgURE 3: Coal mine rescue robot and the related sensor location map. 1, the displacement sensor; 2 , power supply and drive unit; 3 , the control unit; 4, multiparameter detector; and 5, the wind speed and direction sensors.

due to thermal motion of gas molecules, which on the one hand is due to the gas concentration being higher than the concentration of gas in airflow and on the other hand is also affected by temperature difference and ventilation eddy current.

The diffusion motion obeys Fick's law of diffusion; Fick's first law expression is

$$
J=-D \frac{d C}{d x}
$$

where $D$ is gas diffusion coefficient, $C$ is the concentration of gas molecules, $d C / d x$ is concentration gradient, sign "-" indicates a direction opposite to the concentration gradient diffusion direction, indicating that diffusion component is made of high concentration to low concentration diffusion region, and $J$ is diffusion flux. The diffusion coefficient depends on the pressure, temperature, and composition of gas mixture, usually determined by experiment. Document [17] gives the diffusion coefficient by experimental determination as follows:

$$
D=1.103 \times 10^{-2} T^{1.75} \frac{\left(M_{A}+M_{B}\right) /\left(M_{A} M_{B}\right)}{P\left(V_{A}+V_{B}\right)^{2}} .
$$

Among them, $V_{A}$ and $V_{B}$ are the volume of corresponding gas component; $P$ is the gas pressure; $T$ is temperature; $M_{A}$ and $M_{B}$ are the molecular weight of corresponding gas component.

The air in airflow produces the friction and impact on gas, which force the gas moving with the airflow. Assuming the gas concentration $c$, the gas flow of the surface can be calculated by the convection transport principle, namely, amount of gas flux: $\vec{J}=C \vec{\mu}$, where $\vec{J}$ is the gas diffusion flux and $\vec{\mu}$ is the absolute wind speed along each coordinate axis.

Figure 4 shows the coal mine rescue robots walking on the roadway. Joint search and rescue tasks require multiple robots, and each robot needs to probe different regions. The sample data are the gas and related environmental data in the first 20 meters, collected by the coal mine rescue robot; the wind speed, temperature, and gas concentration data of the mine rescue robot on the coal mine roadway are shown in Figure 5. The coal mine rescue robot predicts the gas concentration in the first 10 meters of the coal mine.

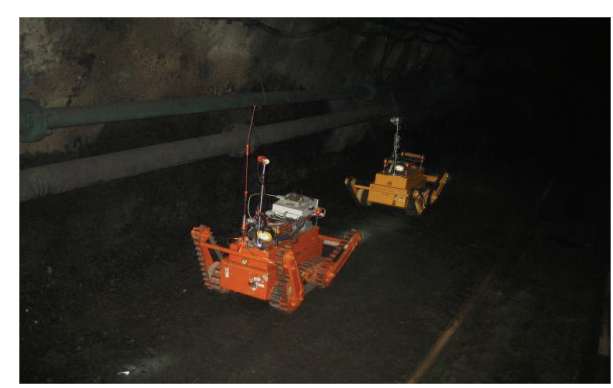

Figure 4: Coal mine rescue robot walking on the roadway.
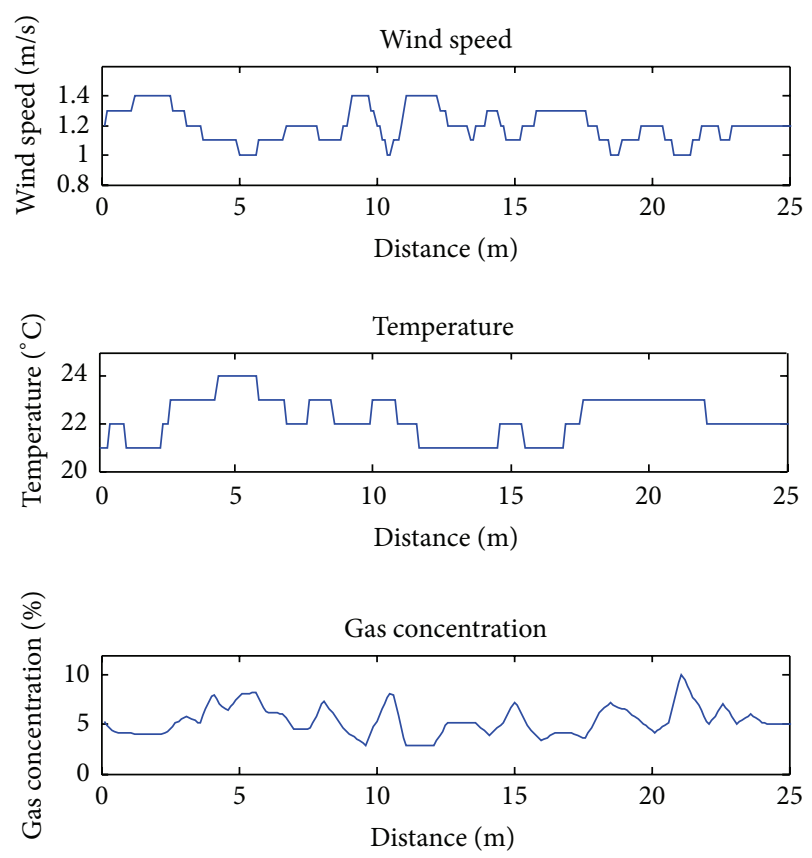

FIGURE 5: Wind speed, temperature, and gas concentration data from the coal mine roadway.

3.2. Experimental Data Processing and Analysis. In this paper, the quantum genetic algorithm is used to optimize the gray neural network to construct the prediction model of the gas concentration in the coal mine underground roadway based on the gas concentration, temperature, and wind speed data collected by the coal mine rescue robots. The construction flow of the forecasting model is shown in Figure 6.

When the coal mine rescue robots predict the gas concentration of the roadway in the coal mine, the main influencing factors are the current position's gas concentration, the ambient temperature, and the wind speed. At the same time, the more the input features, the better the prediction effect of the forecasting model. In general, the greater the wind speed, the more dispersed the gas concentration distribution, and the higher the temperature, the more gathered the gas concentration distribution. The correlations among wind speed, temperature, and gas concentration were analyzed, the correlation coefficients of wind speed with gas concentration and temperature with gas concentration are calculated, and they are shown in Table 4. 


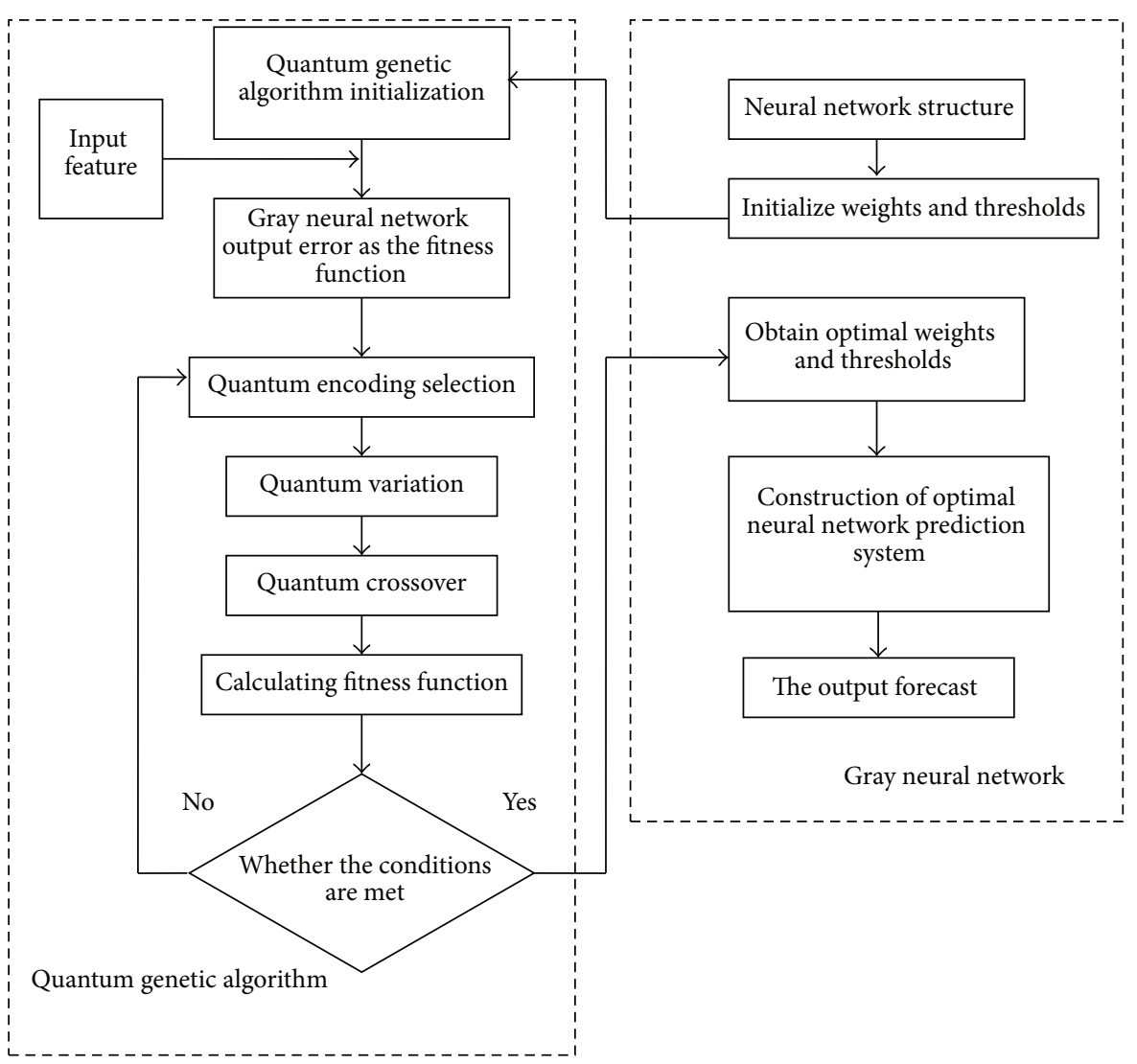

FIGURE 6: Construction flow of the forecasting model.

TABLE 4: The correlations among wind speed, temperature, and gas concentration.

The correlation among wind speed and gas concentration $\quad 0.5701$

The correlation among temperature and gas concentration 0.6774

It can be seen from the calculation results of correlation that the correlation among wind speed and gas concentration belongs to the moderate degree, and the correlation among temperature and gas concentration belongs to strongly correlated degree. Wind speed and temperature are the major factors that affect the distribution of gas concentration.

Therefore, in this paper, the gas concentration of 10 meters is forecasted using the sensor information of the current position and the first meter. The gray neural network is suitable for the small sample and the uncertain system, which has partial information and unknown information and can predict the behavior and laws of the system by the extraction and exploitation of the known information.

A gray neural network was designed in this paper. The LA layer has one neuron, and the LB layer has one neuron. Because of six input vectors, the LC layer has seven neurons, and the output layer LD layer has one neuron. The initial number of training steps of the gray neural network is 100 . The change in the training error of the gray neural network is shown in Figure 7, which shows that, after 6 steps of basic training, the gray neural network converges. The convergence

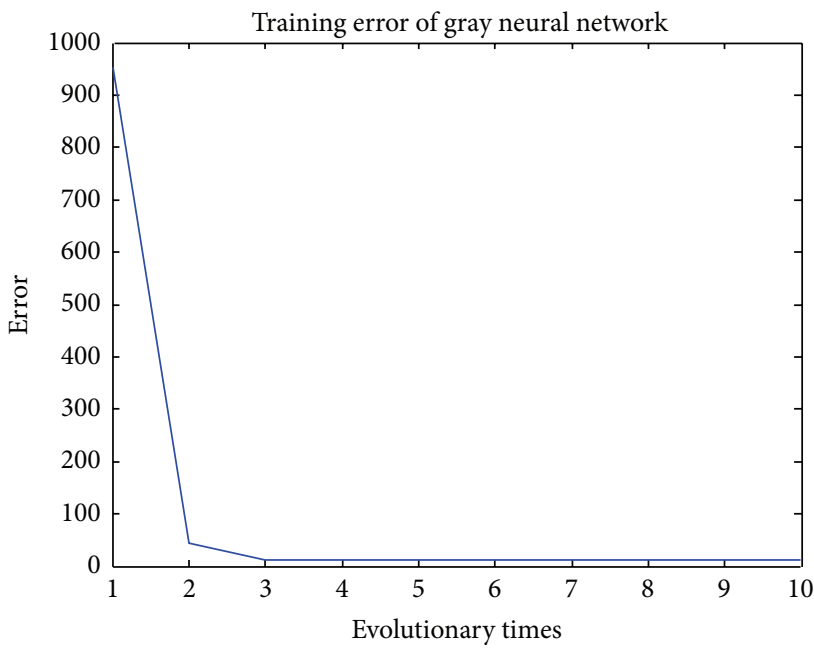

FIgURE 7: The change in the training error of the gray neural network.

rate is fast, but the network is in the local optimum. The weights of the gray neural network cannot be modified. After the gray neural network training is completed, the test data are used for testing. The test data are inputted into the trained gray neural network to predict the gas concentration 10 meters outside the current position of the coal mine rescue 


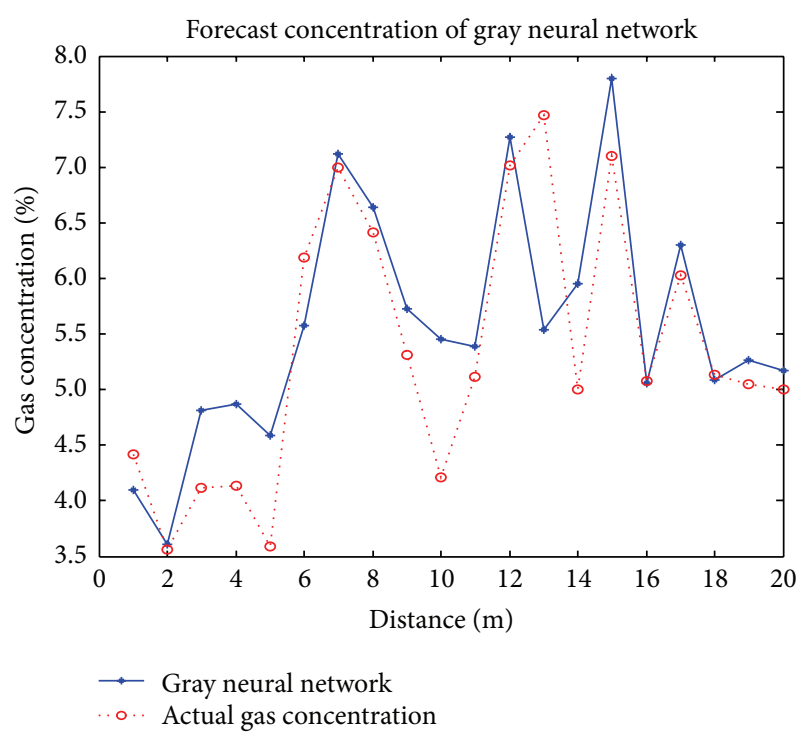

FIGURE 8: Comparison of the gray neural network forecast value and the actual value.

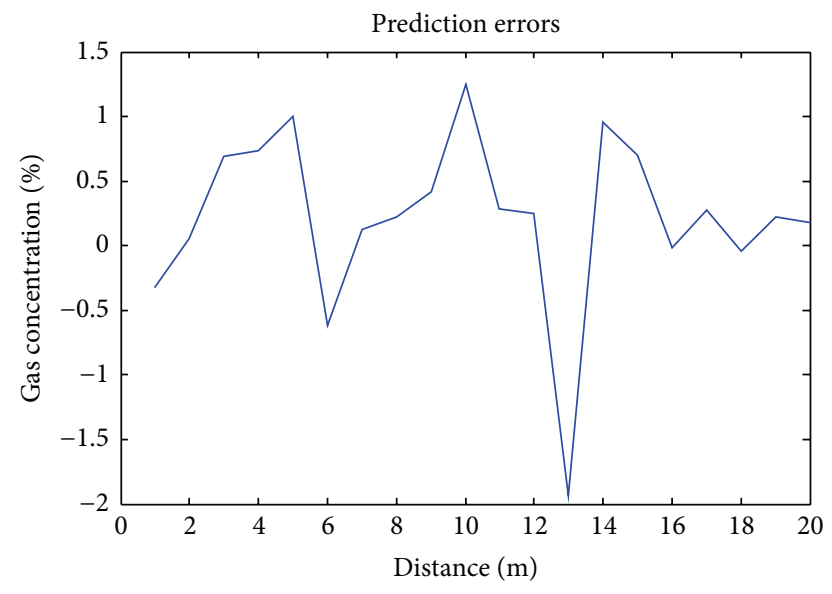

FIGURE 9: The prediction errors of the gray neural network.

robots. Figure 8 compares the gray neural network forecast and the actual gas concentration. Figure 9 is the prediction errors of the gray neural network.

In Figure 8, blue represents the gray neural network forecast and red the actual gas concentration. The gray neural network forecast values are more accurate at a few points, such as $7-8 \mathrm{~m}$ and $16-18 \mathrm{~m}$. For example, the predictive value of gas concentration at $7 \mathrm{~m}$ is 7.137 , while the real value of gas concentration at $7 \mathrm{~m}$ is 7 , so the prediction deviation is 0.137 , and the forecast error is $E_{7}=\left|\left(x^{(1)}(7)-\widehat{x}^{(1)}(7)\right) / \widehat{x}^{(1)}(7)\right|=$ $0.137 / 7.137=1.92 \%$, but at some points, the difference between the predicted value and the actual value is still relatively large, such as $2-5 \mathrm{~m}$ and $9-12 \mathrm{~m}$. For example, the predictive value of gas concentration at $10 \mathrm{~m}$ is 5.5 , while the real value of gas concentration at $10 \mathrm{~m}$ is 4.25 , so the prediction deviation is 1.25 , and the forecast error is $E_{10}=$ $\left|\left(x^{(1)}(10)-\widehat{x}^{(1)}(10)\right) / \widehat{x}^{(1)}(10)\right|=1.25 / 5.5=22.73 \%$, which is significantly higher than the acceptable deviation range.
TABLE 5: Neural network parameter results of the optimization of the quantum genetic algorithm.

\begin{tabular}{lccccccc}
\hline Parameter & $a$ & $b_{1}$ & $b_{2}$ & $b_{3}$ & $b_{4}$ & $b_{5}$ & $b_{6}$ \\
\hline $\begin{array}{l}\text { Parameter } \\
\text { values }\end{array}$ & 0.1476 & 0.3073 & 0.1287 & 0.1485 & 0.1688 & 0.3297 & 0.2620 \\
\hline
\end{tabular}

The overall average error of the gray neural network forecast is $E=(1 / 20) \sum_{i=1}^{20}\left|x^{(1)}(i)-\hat{x}^{(1)}(i)\right| / x^{(1)}(i)=20.37 \%$. The cause of prediction error is various; the main reason has four aspects: First, the prediction error is caused by the measurement error of the gas concentration and sensor used in this paper had already been certified and licensed; second, the prediction error is caused by the selection of predictive factor and the gas concentration is easily affected by gas emission, wind speed, temperature, and concentration of $\mathrm{CO}_{2}$. Due to the small effect of $\mathrm{CO}_{2}$ concentrations, it can be removed by using principal component analysis; therefore, the gas concentration, temperature, and wind speed were been selected as a predictor of gas concentration prediction model; third, adjusting the parameters in the prediction model of grey neural network can improve the convergence speed and stability training error, but how to accurately select the error threshold needs repeat experiments according to the system; fourth, the number of sample datasets will also affect the prediction error; the more the sample data, the better the prediction effect; however, more samples' data means that walking speed of mine rescue robot must be slowed down; this is bound to delay the rescue time and reduce the role of mine rescue robot.

However, because the gray neural network threshold weights are randomly initialized, the network training evolution process can easily fall into the local optimum, and every prediction result is not the same. Being easily trapped in the local minima can be avoided by combining a genetic algorithm with a neural network. The neural network is prone to overfitting and has poor generalization ability; to solve this problem, the quantum genetic algorithm optimizes the parameters $a, b_{1}, b_{2}, b_{3}, b_{4}, b_{5}$, and $b_{6}$ of the neural network. The quantum genetic algorithm uses entity coding and the quantum interference method to train the data of the gray neural network. The error of the gray neural network's prediction value of the training data is used as the fitness function. The population size of the quantum genetic algorithm is 30 , the genetic factor is 7 , and the number of iterations is 70. The optimal individual fitness value of the quantum genetic algorithm changes with the number of iterations, as shown in Figure 10.

Figure 10 shows that the optimal fitness value of the individual fitness gradually tends to be steady after 70 steps of training, and the optimal individual fitness value is 4.29. The gray neural network parameters after training, $a, b_{1}, b_{2}$, $b_{3}, b_{4}, b_{5}$, and $b_{6}$, are shown in Table 5.

The optimal gray neural network parameters are brought into the gray neural network; the test data were inputted into the gray neural network optimized by the quantum genetic algorithm to predict the gas concentration 10 meters outside the current position of the coal mine rescue robots. Figure 11 


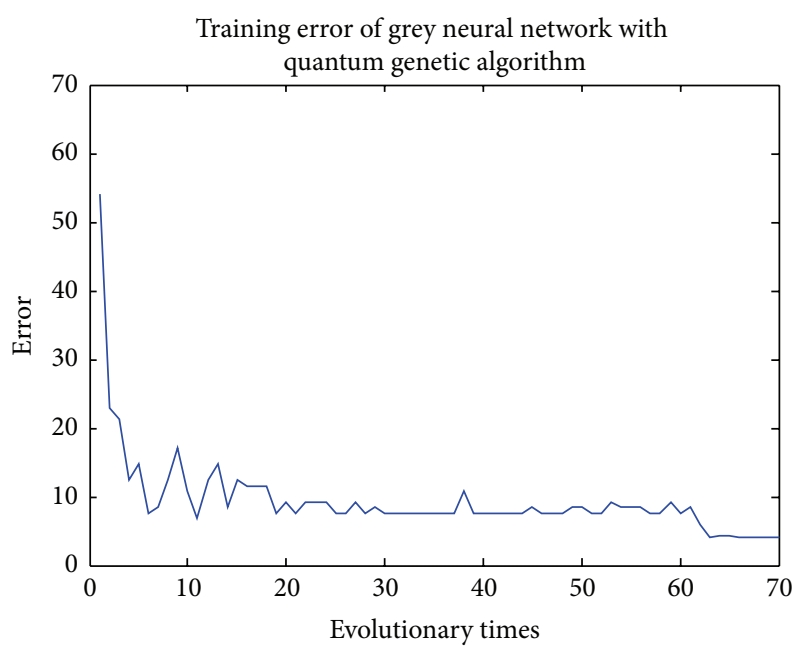

Figure 10: The change in the training error of the gray neural network with quantum genetic algorithm.

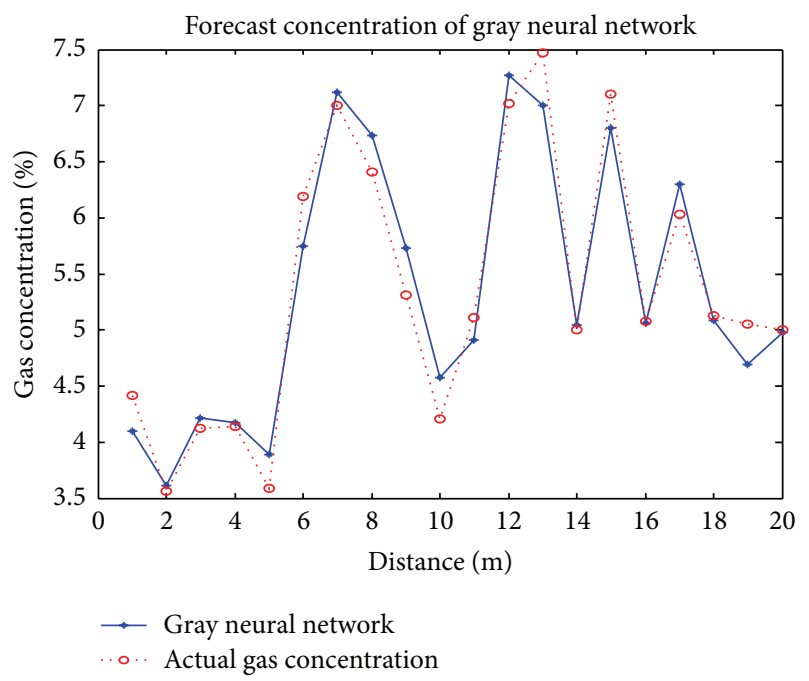

FIGURE 11: Forecast output of the gray neural network optimized by the quantum genetic algorithm.

compares the gray neural network forecast and the actual gas concentration. Figure 12 shows the prediction errors of the gray neural network optimized by the quantum genetic algorithm.

The graph shows that the gas concentration of the gray neural network after the optimization of the quantum genetic algorithm is more accurate. For $5 \mathrm{~m}, 13 \mathrm{~m}, 15 \mathrm{~m}$, and $19 \mathrm{~m}$, the predictive value of gas concentration at $5 \mathrm{~m}$ is 3.96 , while the real value of gas concentration at $5 \mathrm{~m}$ is 3.51 , so the prediction deviation is 0.45 , and the forecast error is $E_{5}=\mid\left(x^{(1)}(5)-\right.$ $\left.\widehat{x}^{(1)}(5)\right) / \widehat{x}^{(1)}(5) \mid=0.45 / 3.96=11.36 \%$, the optimized gray neural network forecast value is close to the actual value, and the overall forecast error is $E=(1 / 20) \sum_{i=1}^{20} \mid x^{(1)}(i)-$ $\widehat{x}^{(1)}(i) \mid / x^{(1)}(i)=9.12 \%$. Compared with the ordinary gray neural network, the overall accuracy gas concentration prediction increases by $(20.37 \%-9.12 \%) / 20.37 \%=55.23 \%$. Therefore, the optimized gray neural network can better

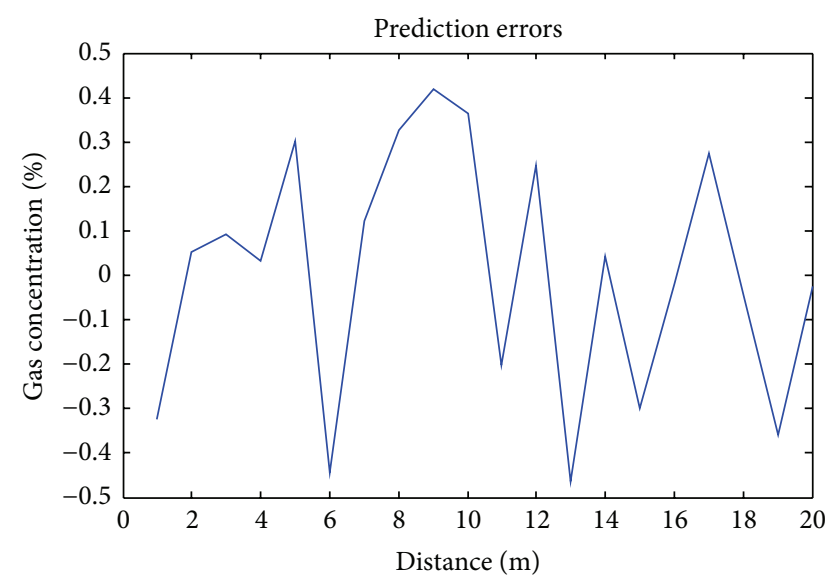

FIGURE 12: Prediction errors of the gray neural network optimized by the quantum genetic algorithm.

help coal mine rescue robots to accurately predict the gas concentration.

\section{Conclusions}

After a coal mine accident, in order for the coal mine rescue robot to successfully execute detection and search and rescue mission, this paper addresses the problem of predicting the roadway gas concentration in front of the coal mine rescue robot based on the real-time data collection of the temperature, wind speed, and gas concentration. The gray neural network cannot fully execute its forecasting capabilities because the gray neural network training can easily fall into the local optimum. The difference between the predicted gas concentration and the actual value is large, and the overall average forecast error is $22.73 \%$. Combining the quantum genetic algorithm and the gray neural network together can solve the shortcomings of the gray neural network and obtain a more accurate prediction of the gas concentration. The experiment shows that the gray neural network optimized by the quantum genetic algorithm is more accurate for predicting the gas concentration. The overall prediction error is $9.12 \%$, and the largest forecasting error is $11.36 \%$. The gas concentration's overall prediction accuracy is improved by $55.23 \%$ compared with the ordinary gray neural network, and predicting the gas concentration of the gray neural network optimized by the quantum genetic algorithm is an effective gas concentration prediction method for the coal mine rescue robot in an underground tunnel.

\section{Competing Interests}

The authors declare that they have no competing interests.

\section{Acknowledgments}

This work is supported by grant of the National 863 Program of China (no. 2012AA041504) and the Policy Guidance Class Plan of Jiangsu Province (no. BY2015024-2). 


\section{References}

[1] A.-R. Zhao, "Prediction method of gas concentration based on wavelet analysis and BP neural network," Coal Mine Machinery, vol. 35, pp. 258-260, 2014.

[2] C. Özgen Karacan, "Modeling and prediction of ventilation methane emissions of U.S. longwall mines using supervised artificial neural networks," International Journal of Coal Geology, vol. 73, no. 3-4, pp. 371-387, 2008.

[3] L. Runqiua, S. Shilianga, W. Aiyoua, L. Wenkea, and Z. Hongpingc, "Research on prediction of gas emission based on selforganizing data mining in coal mines," Procedia Engineering, vol. 84, pp. 779-785, 2014.

[4] X. Hu, S. Yang, X. Zhou, G. Zhang, and B. Xie, "A quantification prediction model of coalbed methane content and its application in Pannan coalfield, Southwest China," Journal of Natural Gas Science and Engineering, vol. 21, pp. 900-906, 2014.

[5] G.-X. Jing, S.-M. Xu, X.-W. Heng, and C.-Q. Li, "Research on the prediction of gas emission quantity in coal mine based on grey system and linear regression for one element," Procedia Engineering, vol. 26, supplement, pp. 1585-1590, 2011.

[6] T. Han, S. Wu, and P. Wang, "Prediction of gas concentration based on residual correction of Markov Chain," Industry and Mine Automation, vol. 40, pp. 28-31, 2014.

[7] S.-G. Cao, Y.-B. Liu, and Y.-P. Wang, "A forecasting and forewarning model for methane hazard in working face of coal mine based on LS-SVM," Journal of China University of Mining and Technology, vol. 18, no. 2, pp. 172-176, 2008.

[8] S. Wei-Hua and Z. Hong-Wei, "Regional prediction of coal and gas outburst hazard based on multi-factor pattern recognition," Procedia Earth and Planetary Science, vol. 1, no. 1, pp. 347-353, 2009.

[9] X.-H. Zhao, G. Wang, K.-K. Zhao, and D.-J. Tan, "On-line least squares support vector machine algorithm in gas prediction," Mining Science and Technology, vol. 19, no. 2, pp. 194-198, 2009.

[10] X. Sun, "Coalmine gas prediction based on fuzzy neural network," Journal of Anhui University of Technology and Science, vol. 29, no. 3, pp. 229-232, 2012.

[11] Y. Liu, Q. Zhao, and W. Hao, "Study of gas concentration prediction based on genetic algorithm and optimizing BP neural network," Mining Safety \& Environmental Protection, vol. 42, pp. 56-60, 2015.

[12] D. Gan and Z. Zhao, "The optimal combination prediction method and its application in gas concentration prediction," Journal of Shandong University of Science and Technology, vol. 31, pp. 55-61, 2012.

[13] R. Guo and G. Xu, "Research on coal mine gas concentration multi-sensor prediction model based on information fusion and GA-SVM," China Safety Science Journal, vol. 23, pp. 33-38, 2013.

[14] J.-Y. Zhang, H. Xu, J. Chen, and X.-D. Cao, "Application research of support vector machine based on particle swarm optimization in gas concentration forecasting," Industry and Mine Automation, vol. 10, pp. 32-35, 2010.

[15] A. O. Caldeira and A. J. Leggett, "Quantum tunnelling in a dissipative system," Annals of Physics, vol. 149, no. 2, pp. 374456, 1983.

[16] S.-Y. Yang, F. Liu, and L.-C. Jiao, "The quantum evolutionary strategies," Acta Electronica Sinica, vol. 29, no. 12, pp. 1873-1877, 1873.
[17] E. N. Fuller, P. D. Schettler, and J. C. Giddings, "New method for prediction of binary gas-phase diffusion coefficients," Industrial \& Engineering Chemistry, vol. 58, no. 5, pp. 18-27, 1966. 


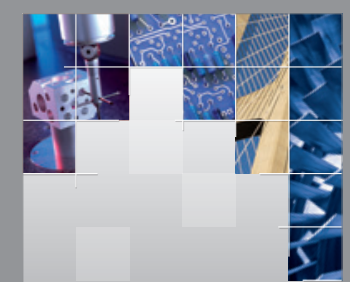

\section{Enfincering}
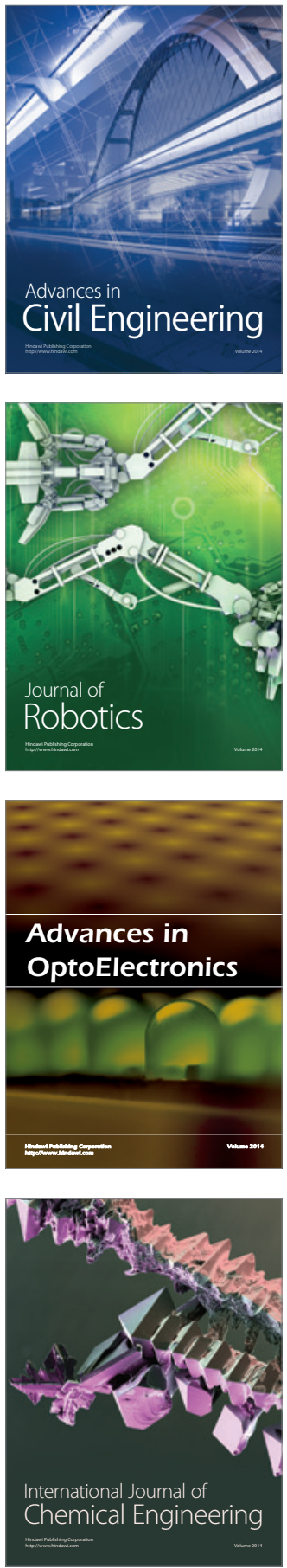

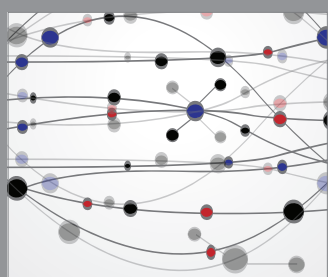

The Scientific World Journal

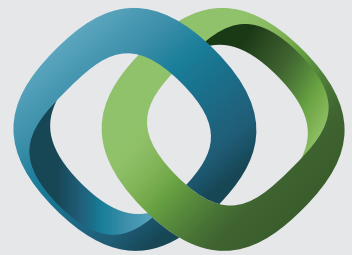

\section{Hindawi}

Submit your manuscripts at

http://www.hindawi.com
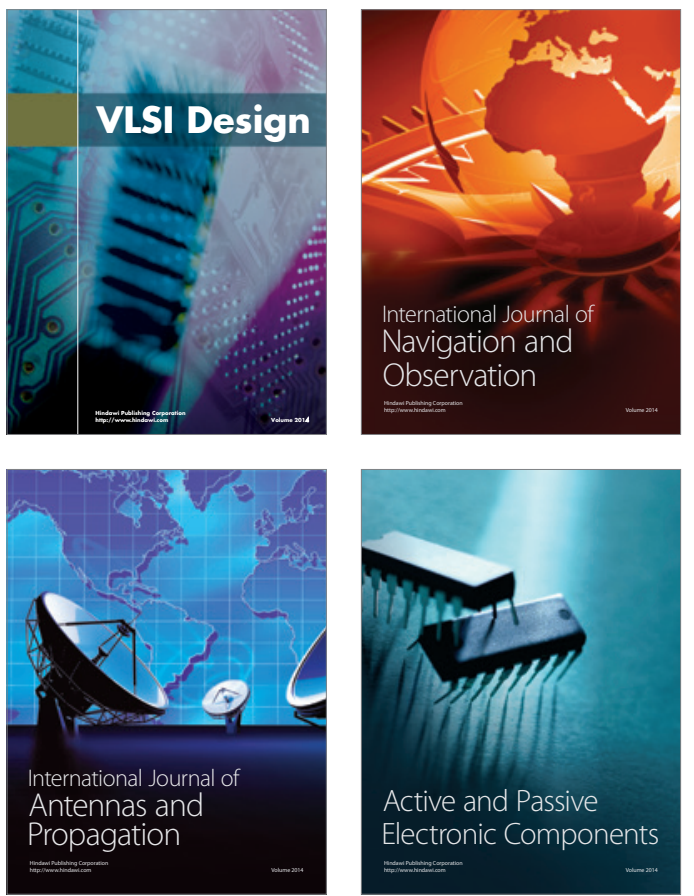
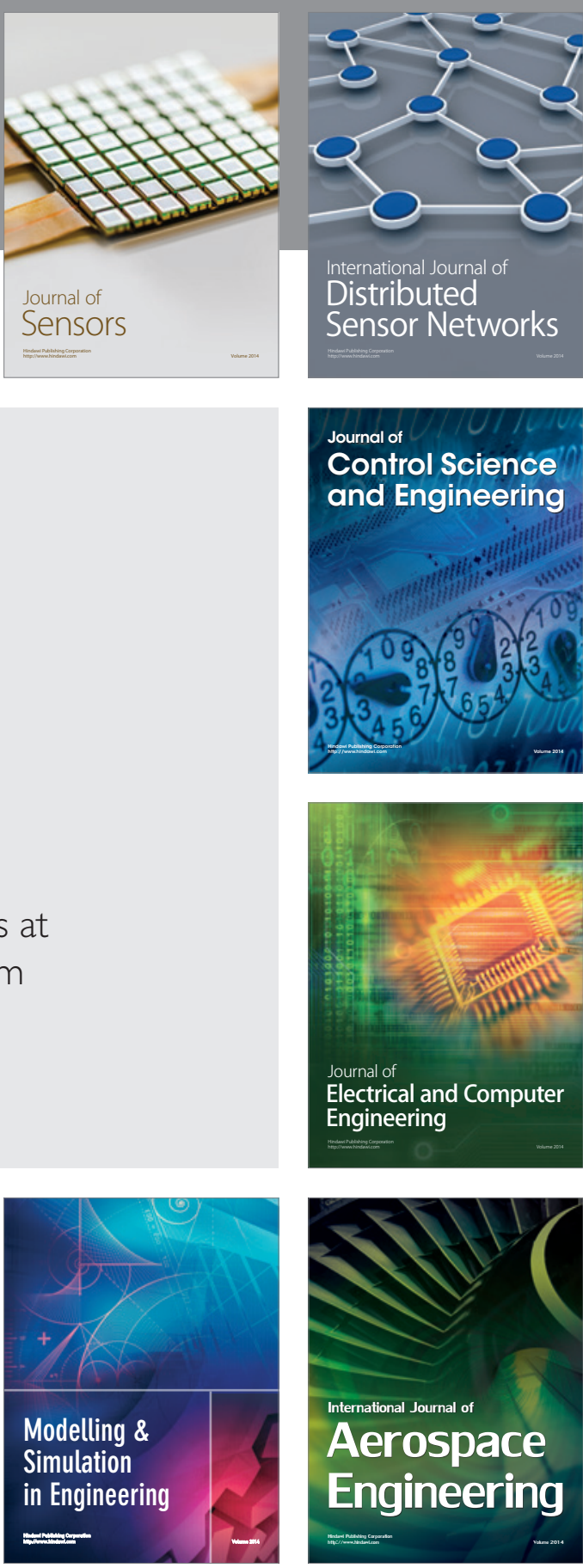

International Journal of

Distributed

Sensor Networks

Journal of

Control Science

and Engineering
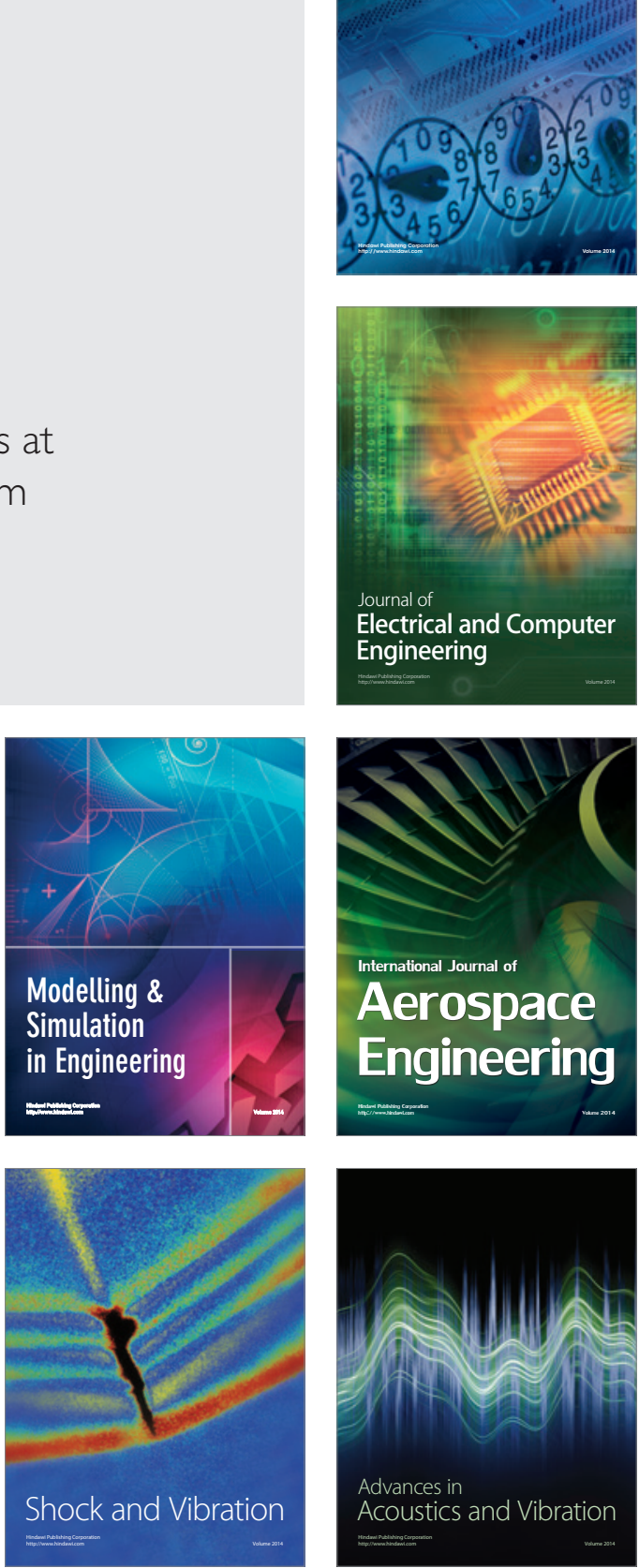